\title{
Electromagnetic radiation initiated by hadronic jets from microquasars in the ISM
}

\author{
V. Bosch-Ramon ${ }^{1}$, F. A. Aharonian ${ }^{2}$, and J. M. Paredes ${ }^{1}$ \\ 1 Departament d'Astronomia i Meteorologia, Universitat de Barcelona, Av. Diagonal 647, 08028 Barcelona, Spain \\ e-mail: vbosch@am.ub.es \\ 2 Max-Planck-Institut fur Kernphysik, Saupfercheckweg 1, Heidelberg, 69117, Germany
}

Received 4 August 2004 / Accepted 2 November 2004

\begin{abstract}
Microquasars are potential candidates to produce a non-negligible fraction of the observed galactic cosmic rays. The protons accelerated at the jet termination shock interact with the interstellar medium and may produce detectable fluxes of extended emission at different energy bands: high-energy and very high-energy $\gamma$-rays produced by neutral pion-decay, synchrotron and bremsstrahlung emission in a wide energy range generated by the secondary electrons produced by charged pion-decay. We discuss the association between this scenario and some of the unidentified EGRET sources in the galactic plane.
\end{abstract}

Key words. X-rays: binaries - ISM: general - gamma-rays: observations - gamma-rays: theory

\section{Introduction}

The high-energy $\gamma$-ray instrument EGRET detected about 170 sources that still remain unidentified. Among them, an important fraction are non-variable galactic sources. Several astrophysical objects could explain the nature of this set of steady galactic $\gamma$-ray sources: pulsars, supernova remnants, molecular clouds, etc. (see Torres et al. 2003; and also Nolan et al. 2003). However, new types of galactic sources could be also the counterparts of this $\gamma$-ray steady emission detected by EGRET. In particular, microquasars (MQs) have turned out to be likely sources of $\gamma$-rays (Paredes et al. 2000), and they could be indirect sources of non-variable (at EGRET timescales) $\gamma$-ray emission too. Indeed, these objects present powerful jets that show persistent or transient activity and can carry huge amounts of kinetic energy during the life of the MQ (millions of years for high-mass MQs and longer lifetimes for low-mass ones, Tauris \& Van den Heuvel 2003). Following the standard model for jet formation in MQs (i.e. Meier 2003), the jets of microquasars transport a hadronic component extracted from the accretion disk, which is formed by the matter accreted from the companion star. Once the jet terminates, two different scenarios are possible: a smooth transition jet-interstellar medium (ISM), where jet protons would be released in the ISM with velocities similar to the original jet velocity, or a shock-like interaction with the ISM, where jet protons would be accelerated by the Fermi mechanism. If the first possibility occurs, these particles could be a minor but non-negligible part of the cosmic rays produced in our Galaxy at low energies (about GeV energies). Moreover, in the second case, protons could reach higher energies, depending on the conditions within the jet-ISM shock region (for an extended discussion, see Heinz \& Sunyaev 2002). From electrodynamical arguments (see, i.e., Aharonian et al. 2002), if the magnetic field and the size of the final part of the jet is similar to that, for instance, considered in Paredes et al. (2002), proton energies of about $10^{5} \mathrm{GeV}$ may be achieved. Assuming that a non-thermal population of high energy protons is released from the terminal part of a jet, these particles will diffuse through the interstellar medium. At certain distances from the accelerator, the protons can interact with high-density regions (i.e. molecular clouds), producing extended and steady emission at different energies. Due to propagation effects, the outcomes from such interactions can differ strongly depending on the age, the nature (impulsive or continuous) of the accelerator and the distance between the accelerator and the cloud (see Aharonian \& Atoyan 1996). It should be noted that hadronic $\gamma$-rays could be produced at much smaller scales as well, e.g. at interaction of the relativistic jet of the compact object with the dense wind of the stellar companion, as suggested by Romero et al. (2003).

In this work, we explore the possibility that MQs could initiate indirect and steady sources of $\gamma$-rays and lower frequency radiation throughout interactions between high energy protons released from their jets and nearby molecular clouds. We have developed a time-dependent model that calculates the broadband spectrum of the emission coming out from the pp primary interactions as well as the emission produced by the secondary particles (electrons and positrons) created during the first process. This model takes into account the propagation effects due to both energy-dependent diffusion and the pp interaction energy losses of protons during their travel from the accelerator to the cloud. From this point, when referring to an impulsive MQ, 
we mean a transient jet of MQ interacting with a cloud, and by a continuous MQ, we mean the same but for a persistent jet of a MQ.

\section{MQ-cloud interactions and production of $\gamma$-rays}

Jets of MQs must end somewhere, although the details of this are still unclear (Heinz 2002). Some MQs present persistent jets that emit at different energy bands up to hundreds of $\mathrm{AU}$ with typical jet kinetic energy luminosities from $10^{37}$ to $10^{39} \mathrm{erg} \mathrm{s}^{-1}$ (LS 5039, Ribó 2002; SS 433, Marshall et al. 2002; Spencer 1984). Other members belonging to this type of galactic jet sources present very powerful transient ejections that can extend larger distances and contain several orders of magnitude more jet kinetic energy than the persistent cases (i.e. GRS 1915+105, Mirabel \& Rodriguez 1994; Cygnus X-3, Martí et al. 2000). Finally, some MQs present extended structures at radio frequencies (1E 1740.7-2942, Mirabel \& Rodríguez 1999) or at X-rays (XTE J1550-564, Corbel et al. 2002; Kaaret et al. 2003) at scales of 0.1-1 pc, which could be related to acceleration of particles of a jet interacting with the environment (i.e. Wang et al. 2003).

Below, we initially assume that the jet has a significant population of protons. These protons can be cold, or cooled during their propagation through the jet due to, i.e., adiabatic losses. They can be cold in the reference frame of the jet, but in the frame of the ISM their energy could be as large as $\Gamma m_{\mathrm{p}} c^{2}$, where $\Gamma$ is the Lorentz factor of the jet and $m_{\mathrm{p}}$ is the proton mass. However, they can also be accelerated in the terminal part of the jet owing to shocks between the matter of the jet and the ISM. Therefore, a population of non-thermal protons can extend up to very high energies, and these particles are released since the low magnetic field strength is not enough to keep them within the accelerator. Once the particles break free, they diffuse in the ISM with a diffusion speed depending on their energy and, close to or further from the releasing point, the protons interact with higher density regions. The interaction between the high energy protons and the interstellar hydrogen nuclei will produce charged and neutral pions $\left(\pi^{-}, \pi^{+}\right.$and $\left.\pi^{0}\right)$; the first will decay to electrons and positrons and the second to photons. The primary radiation, $\pi^{0}$-decay photons, is in the $\gamma$-ray band, but the secondary particles can produce significant fluxes of synchrotron (from radio frequencies to X-rays) and bremsstrahlung emission (from soft $\gamma$-rays to $\mathrm{TeV}$ range), and generally with less efficiency, inverse Compton (IC) emission through interaction with the ambient infrared (IR) photons.

\subsection{Evolution in time and distance of the proton energy distribution}

To calculate the emissivity of a source due to different mechanisms, we need to know first what is the energy distribution of the high energy protons at different times and distances from the acceleration site. Therefore, we have assumed that the (initial) energy distribution of the accelerated protons follows a power-law plus a high-energy cutoff:

$$
f_{\mathrm{p} 0}\left(E_{\mathrm{p}}\right)=K E_{\mathrm{p}}^{-p} \exp \left(-E_{\mathrm{p}} / E_{\mathrm{pmax}}\right),
$$

where $K$ is the normalization constant of the function (to compare with the total number of particles or total energy contained by them), $E_{\mathrm{p}}$ is the proton energy and $E_{\mathrm{pmax}}$ is the energy where the exponentical cut-off starts to dominate over the power-law. $E_{\text {pmax }}$ is determined by the efficiency of the accelerator and will be treated here as a parameter, avoiding detailed treatments of the acceleration region.

To determine the energy distribution of protons in distance and time $\left(f_{\mathrm{p}}\left(E_{\mathrm{p}}, R, t\right)\right)$, we have used the solution found by Atoyan et al. (1995) to the diffusion equation (Ginzburg \& Syrovatskii 1964) in the spherically symmetric case:

$\frac{\partial f_{\mathrm{p}}}{\partial t}=\frac{D}{R^{2}} \frac{\partial}{\partial R}\left[R^{2} \frac{\partial f_{\mathrm{p}}}{\partial R}\right]+\frac{\partial}{\partial E_{\mathrm{p}}}(P f)+Q$.

Here, $t$ is the source age, $R$ the distance to the source of protons, $D$ is the diffusion coefficient, which is assumed as $D\left(E_{\mathrm{p}}\right) \propto E_{\mathrm{p}}^{\chi}$, $P$ is the continuous energy loss rate $\left(P=-\mathrm{d} E_{\mathrm{p}} / \mathrm{d} t\right)$ and $Q$ is the source function, such that $Q\left(E_{\mathrm{p}}, R, t\right)=f_{\mathrm{p} 0}\left(E_{\mathrm{p}}\right) \delta(\boldsymbol{R}) \delta(t)$. $\delta(\boldsymbol{R})$ and $\delta(t)$ are delta functions in space and time, respectively. To account for the energy losses during the diffusive transport of protons, since the dominant cause of loss is nuclear interactions, we have $P\left(E_{\mathrm{p}}\right)=E_{\mathrm{p}} / \tau_{\mathrm{pp}}$, where $\tau_{\mathrm{pp}} \approx$ $6 \times 10^{7}\left(n / 1 \mathrm{~cm}^{-3}\right)^{-1} \mathrm{yr}$, and $n$ is the hydrogen density of the medium. We assume an acceleration spectrum constant in time given by Eq. (1).

In our particular case of a power-law plus a high-energy cutoff injection spectrum, and a diffusion coefficient that depends on energy following a power-law, the general solution is reduced to:

$$
\begin{aligned}
f_{\mathrm{p}}\left(E_{\mathrm{p}}, R, t\right) \approx & \frac{K E_{\mathrm{p}}^{-p} \exp \left(-E_{\mathrm{p}} / E_{\mathrm{pmax}}\right)}{\pi^{3 / 2} R_{\mathrm{dif}}^{3}} \\
& \times \exp \left(-\frac{(p-1) t}{\tau_{\mathrm{pp}}}-\frac{R^{2}}{R_{\mathrm{dif}}^{2}}\right),
\end{aligned}
$$

where $R_{\text {dif }}$ is the diffusion radius and corresponds to the radius of the sphere up to which the particles of energy $E_{\mathrm{p}}$ effectively propagate during the time $t$ after they left the acceleration site,

$R_{\mathrm{dif}}\left(E_{\mathrm{p}}, t\right)=2\left(D\left(E_{\mathrm{p}}\right) t \frac{\exp \left(t \chi / \tau_{\mathrm{pp}}\right)-1}{t \chi / \tau_{\mathrm{pp}}}\right)^{1 / 2}$.

$D\left(E_{\mathrm{p}}\right)=D_{10}\left(E_{\mathrm{p}} / 10 \mathrm{GeV}\right)^{\chi}$, and $D_{10}$ is the diffusion coefficient normalization constant in the ISM. For further details regarding diffusion of particles in the ISM, see Aharonian \& Atoyan (1996).

From Eq. (3) we can determine the proton energy distribution at any $t$ and $R$, starting with an impulsively injected population of particles (Eq. (1)). To model the energy distribution of protons for a continuous MQ, we will need to integrate in time from the initial to the present time, taking into account that the source function represents the number of released particles per energy and time units, and not the number of released particles per energy unit, as in the impulsive case.

\subsection{Emission from interacting protons and hydrogen nuclei}

We calculate the radiative effects of diffusing high energy protons that penetrate a high density region of the ISM. We will 
Table 1. Adopted parameter values.

\begin{tabular}{lcc}
\hline \hline Parameter & Symbol & Value \\
\hline Diffusion coefficient normalization constant & $D_{10}$ & $10^{27} \mathrm{~cm}^{2} \mathrm{~s}^{-1}$ \\
Diffusion power-law index & $\chi$ & 0.5 \\
ISM medium density & $n$ & $0.1 \mathrm{~cm}^{-3}$ \\
High density ISM region/cloud density & $n_{\mathrm{H}}$ & $10^{4} \mathrm{~cm}^{-3}$ \\
Mass of the high density ISM region/cloud & $M$ & $5 \times 10^{3} M_{\odot}$ \\
Magnetic field within the cloud & $B$ & $5 \times 10^{-4} \mathrm{G}$ \\
IR radiation energy density within the cloud & $U$ & $10 \mathrm{eV} \mathrm{cm}^{-3}$ \\
Planckian grey body temperature (IR) & $T$ & $50 \mathrm{~K}$ \\
Power-law index of the high energy protons & $p$ & 2 \\
Cut-off energy of the high energy protons & $E_{\mathrm{pmax}}$ & $10^{5} \mathrm{GeV}$ \\
Kinetic energy luminosity for accelerated protons in the continuos MQ & $L_{\mathrm{k}}$ & $10^{37} \mathrm{erg} \mathrm{s}^{-1}$ \\
Kinetic energy for accelerated protons in the impulsive MQ & $E_{\mathrm{k}}$ & $10^{48} \mathrm{erg}$ \\
\hline
\end{tabular}

assume that the channels of pp interaction that yield either $\pi^{0}$, $\pi^{-}$or $\pi^{+}$are roughly equiprobable, thus the functions that represent the photon, the electron and the positron energy distributions resulting from the pion decay will be taken to be similar (in the relativistic regime of secondary particles).

The emissivity of the photons produced by $\pi^{0}$-decay $\left(q_{\gamma}\right)$ at given $R$ and $t$ can be calculated through Eq. (5):

$q_{\gamma}\left(E_{\gamma}, R, t\right)=2 \int_{E_{\pi \min }}^{E_{\pi \max }} \frac{q_{\pi}\left(E_{\pi}, R, t\right)}{\sqrt{E_{\pi}^{2}-m_{\pi}^{2} c^{4}}} \mathrm{~d} E_{\pi}$,

where $E_{\gamma}$ and $E_{\pi}$ are the energy of the emitted photon and the decaying pion respectively, $E_{\pi \min }=E_{\gamma}+m_{\pi}^{2} c^{4} / 4 E_{\gamma}, m_{\pi}$ is the pion mass and $c$ is the speed of light. The neutral pion emissivity from pp interaction $\left(q_{\pi}\right)$ can be found through Eq. (6):

$$
\begin{aligned}
q_{\pi}\left(E_{\pi}, R, t\right)= & c n_{\mathrm{H}} \int_{E_{\mathrm{p} \min }}^{E_{\mathrm{pmax}}} \delta\left(E_{\pi}-k_{\pi} E_{\mathrm{kin}}\right) \\
& \times \sigma_{\mathrm{pp}}\left(E_{\mathrm{p}}\right) n_{\mathrm{p}} f_{\mathrm{p}}\left(E_{\mathrm{p}}, R, t\right) \mathrm{d} E_{\mathrm{p}} \\
= & \frac{c n_{\mathrm{H}}}{k_{\pi}} \sigma_{\mathrm{pp}}\left(m_{\mathrm{p}} c^{2}+E_{\pi} / k_{\pi}\right) f_{\mathrm{p}}\left(m_{\mathrm{p}} c^{2}+E_{\pi} / k_{\pi}, R, t\right),
\end{aligned}
$$

where $k_{\pi}$ is the mean fraction of the kinetic energy $\left(E_{\mathrm{kin}}=E_{\mathrm{p}}-m_{\mathrm{p}} c^{2}\right)$ of the proton transferred to the secondary pion per collision, $n_{\mathrm{H}}$ is the density of particles of the high density ISM region, and $\sigma_{\mathrm{pp}}\left(E_{\mathrm{p}}\right)$ is the cross section of the pp interaction. This approach of emissivity calculation of $\pi^{0}$-decay $\gamma$-rays gives quite good accuracy down to energies $\sim 1 \mathrm{GeV}$ (see Aharonian \& Atoyan 2000).

Once we have obtained the emissivity, we need only to multiply by the total volume of the interaction region $(V)$ to find the total number of photons generated per energy unit and second. We suppose that the cloud is homogeneous. Also, its size must be small enough compared to $R$ to assume the same energy distribution for the high energy protons everywhere in the interaction region. Actually, one can assume that for a high density region with size smaller than (but similar to) $R$, the changes in the proton spectrum are not significant enough to be taken into account at our level of approximation. Moreover, for the involved timescales, protons do not interact several times within the cloud, because the pp interaction cooling time is longer than the escape time in the proton energy range relevant here. Then, the Spectral Energy Distribution (SED) of the emitted $\gamma$-rays due to the pp interaction within a cloud at a given $t$ (age of the proton source) and $R$ (distance from the proton source to the cloud) is:

$E_{\gamma} L\left(E_{\gamma}\right)=E_{\gamma}^{2} V q_{\gamma}\left(E_{\gamma}, R, t\right)$

The number of electrons per time and energy units produced within a cloud at a certain $R$ and $t\left(Q_{\mathrm{e}}\left(E_{\mathrm{e}}, R, t\right)\right)$ presents a similar distribution, where $E_{\mathrm{e}}$ is the electron energy.

\subsection{Secondary particles and their emission}

We have introduced bremsstrahlung, synchrotron and IC energy losses for the secondary leptons in order to get the proper electron energy distribution. Also, we have assumed that leptons remain within the cloud after their creation. This is a reasonable assumption as a first order approach for electrons emitting via synchrotron process at frequencies above $10 \mathrm{GHz}$, taking into account that the cooling time of those electrons within the cloud is shorter than their escape time, assuming a diffusion for electrons inside similar to the one for protons outside. We note also that for sources younger than $\sim 10^{4} \mathrm{yr}$, secondaries emitting roughly below $10 \mathrm{GHz}$ have not had enough time to escape yet. We will not study emission at lower energies, since this requires a more complex approximation, which will be performed in future work. We have determined the dependence on time of the electron energy adopting quite reasonable assumptions: homogeneous and isotropic velocity distribution of electrons, a homogeneous distribution of hydrogen nuclei, and constant magnetic and radiation fields ( $B$ and $U$ respectively). Regarding radiation fields, we have taken into account the $2.7 \mathrm{~K}$ photon galactic background plus an additional $50 \mathrm{~K}$ photon field considered to be produced inside the cloud (see Table 1). These IC losses, although unavoidable, are not significant unless very high density IR fields are present, which has not been excluded (see Sect. 3.7). The electron energy is given by:

$E_{\mathrm{e}}(t)=\frac{a_{0} n_{\mathrm{H}} E_{\mathrm{e} 0}}{\left(a_{0} n_{\mathrm{H}}+E_{\mathrm{e} 0} C\right) \exp \left[a_{0} n_{\mathrm{H}}\left(t-t_{0}\right)\right]-E_{\mathrm{e} 0} C}$, 
where $C=\left(a_{\mathrm{s}} B^{2}+a_{\mathrm{c}} U\right), a_{0}=8.1 \times 10^{-16}, a_{\mathrm{s}}=2.37 \times 10^{-3}$ and $a_{\mathrm{c}}=3.97 \times 10^{-2}$ in cgs units. Here, $a_{0}$ is related to the bremsstrahlung losses, and $a_{\mathrm{s}}$ and $a_{\mathrm{c}}$ are associated with the synchrotron and the IC losses (Thomson regime) respectively. $t_{0}$ is the initial time, corresponding to an electron energy $E_{\mathrm{e} 0}$.

From $E_{\mathrm{e}}(t)$ and the continuity equation we can predict how the injected secondary particles, following an initial $Q_{\mathrm{e}}$, will change their energy distribution in time. Knowing how an injected $Q_{\mathrm{e}}$ evolves in time, we integrate the different evolved values of $Q_{\mathrm{e}}$ from the initial time to the present moment. Doing so, we get the total number of electrons per energy unit at any $t$ and $R: N_{\mathrm{e}}\left(E_{\mathrm{e}}, R, t\right)$.

We can compute $N_{\mathrm{e}}\left(E_{\mathrm{e}}, R, t\right)$ using numerical calculations for any value of $E_{\mathrm{e}}, R$ and $t$. Knowing the previous function, the magnetic and radiation fields, and the density of the cloud, one can easily calculate the emission from these secondary particles produced via synchrotron radiation, bremsstrahlung and IC scattering (i.e. Blumenthal \& Gould 1970).

\section{Performance of the model for different cases}

We go through different cases that might be relevant. The adopted parameter values are presented in Table 1, although some of them could change depending on the particular scenario. We have computed the emission produced by four different radiative processes: $\pi^{0}$-decay, synchrotron radiation, bremsstrahlung and IC scattering. For the impulsive case, we have considered a very active source, $L_{\mathrm{k}} \sim 10^{39} \mathrm{erg} \mathrm{s}^{-1}$, but operating a short period of time, $\sim 30 \mathrm{yr}$, which implies a $E_{\mathrm{k}} \sim 10^{48} \mathrm{erg}$ (transfered to the accelerated protons). We note that this value for the kinetic energy luminosity of protons is average during the impulsive phase, and it takes into account periods of less activity and recurrent super-Eddington accretion bursts within these $30 \mathrm{yr}$. For a continuous MQ, we have adopted moderate kinetic energy luminosities for the jet (transfered to the accelerated protons) of about $10^{37} \mathrm{erg} \mathrm{s}^{-1}$ (see Table 1).

In the case of impulsive MQ, as is mentioned in Sect. 2 for the case of SS 433, we could have a persistent jet presenting a kinetic energy luminosity similar to or even more than $L_{\mathrm{k}} \sim 10^{39} \mathrm{erg} \mathrm{s}^{-1}$ (Marshall et al. 2002, Spencer 1984). It is a super-Eddington accretion system (as can be seen from the huge amount of jet kinetic energy ), but the ejection appears to be persistent. It could also be the case that a microquasar suffering recurrent or periodic outbursts delivered, on average, the amount of proton kinetic energy assumed here. For instance, GRS 1915+105, which can easily reach superEddington accretion rates, presents large outbursts like the one in 1994 (Mirabel \& Rodriguez 1994), with kinetic energy luminosities close to $10^{41} \mathrm{erg} \mathrm{s}^{-1}$ (Gliozzi et al. 1999). Otherwise, for a continuous MQ, assuming a proton acceleration efficiency of $10 \%$ and a jet carrying $20 \%$ of the accretion power, an accretion rate of $10^{-8} M_{\odot} \mathrm{yr}^{-1}$ is required. Therefore, the energetic assumptions of this work are not rare for microquasars.

Regarding $p$, we have adopted a value of 2 . Cosmic ray spectrum is assumed to be injected with power-law indices of around 2.3. However, from indirect spectral considerations of radiation emitted from the jets of MQs (i.e. their electron

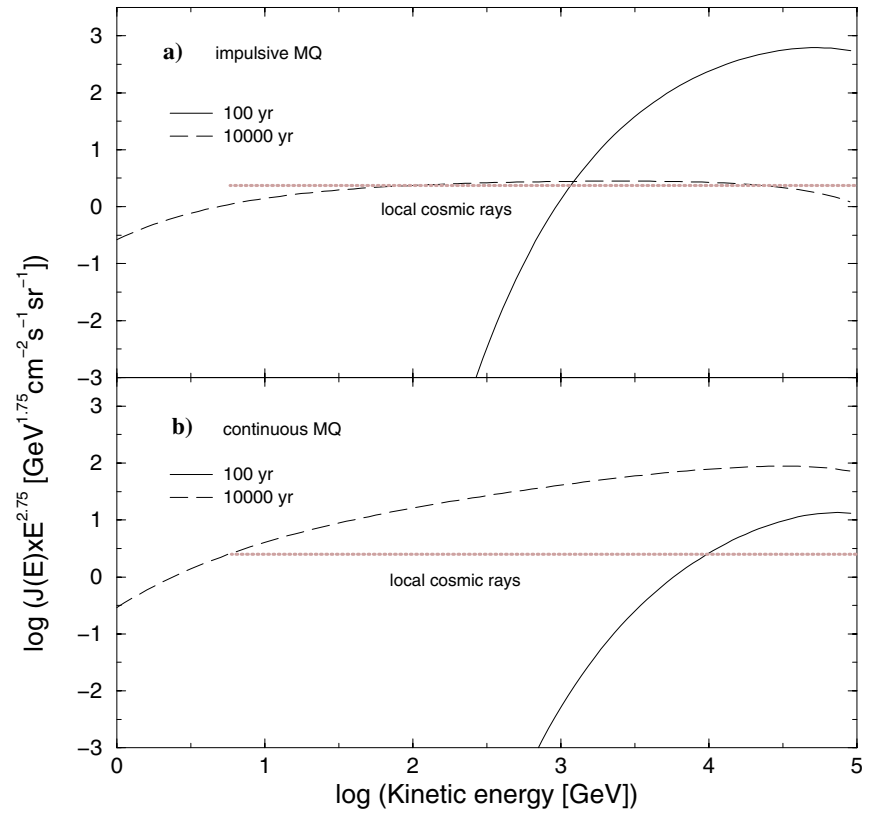

Fig. 1. Spectrum of protons released from a source at $R=10 \mathrm{pc}$ after $t=100 \mathrm{yr}$ (solid line) and $10000 \mathrm{yr}$ (long-dashed line). The dotted horizontal line shows the flux of cosmic ray protons observed near the Earth. a) For an impulsive MQ. b) For a continuous MQ.

spectrum inferred from synchrotron emission), it seems more suitable in that case to take a slightly harder value. The different explored situations are presented in the next subsections. For each case, the relevant paramater values are indicated in italic.

\subsection{Proton flux evolution}

$R=10 \mathrm{pc} ; t=100,10000 \mathrm{yr}$.

We have computed the proton spectra $\left(J\left(E_{\mathrm{p}}\right) \times E^{2.75}\right)$ for an impulsive and a continuous MQ together with the cosmic ray spectrum. The results are plotted in Fig. 1. We note a harder spectrum and more particles for a continuous MQ compared to an impulsive MQ at older ages. This is due to the continuous particle injection from the jet of the MQ in the first case.

\subsection{Proton and electron energy distributions}

$R=10 \mathrm{pc} ; t=100,1000 \mathrm{yr}$.

We have computed the proton and the electron energy distributions $\left(J\left(E_{\mathrm{p} / \mathrm{e}}\right) \times \frac{4 \pi}{c} V\right)$ within a cloud, plotting them together in the same graph to demonstrate their evolution with time. The impulsive and the continuous cases are shown in Figs. 2 and 3 respectively. We note that, from the diffusion radius (Eq. (4)) and the time-dependent electron energy (Eq. (8)), we can recognize the peaks and turning points of both distribution functions at those energies that correspond roughly to the protons and electrons that dominate at that time. Another interesting feature of Figs. 2 and 3 is that the number of protons and electrons per volume and energy units in the continuous case moves closer to the number in the impulsive case as $t$ is increased, since for the continuous source the protons and electrons accumulate (accumulate for protons) in the cloud with a continuous 


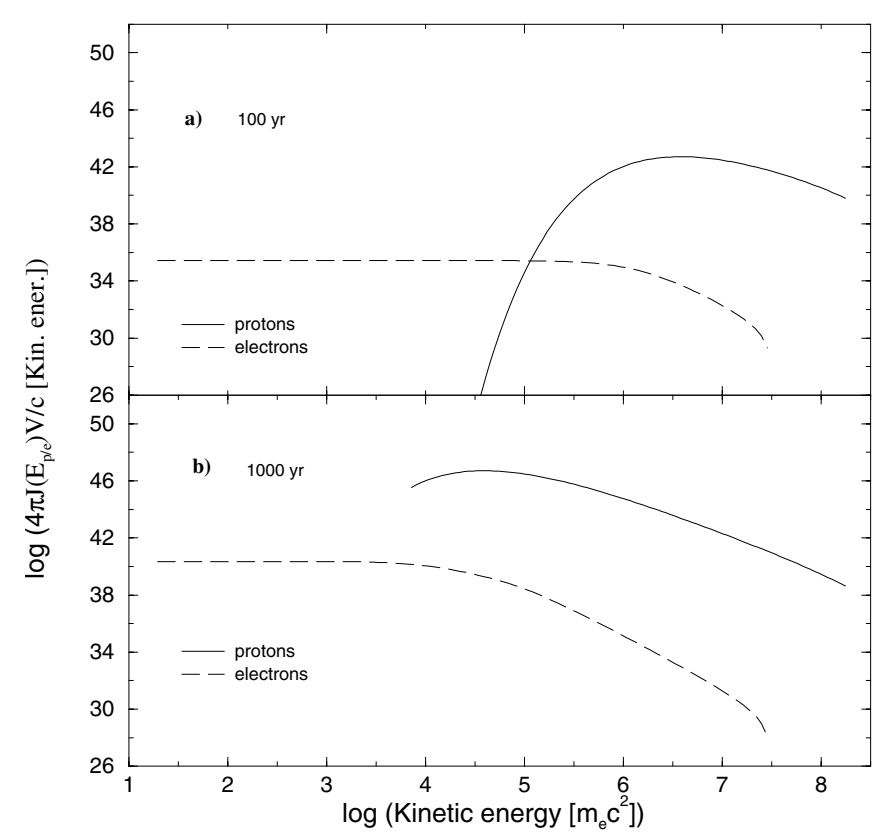

Fig. 2. Proton and electron energy distributions (solid and long-dashed lines respectively) for an impulsive MQ at $R=10 \mathrm{pc}$. The kinetic energy axis units are $\mathrm{m}_{\mathrm{e}} \mathrm{c}^{2}$. a) The source age is $t=100 \mathrm{yr}$. b) The source age is $1000 \mathrm{yr}$.

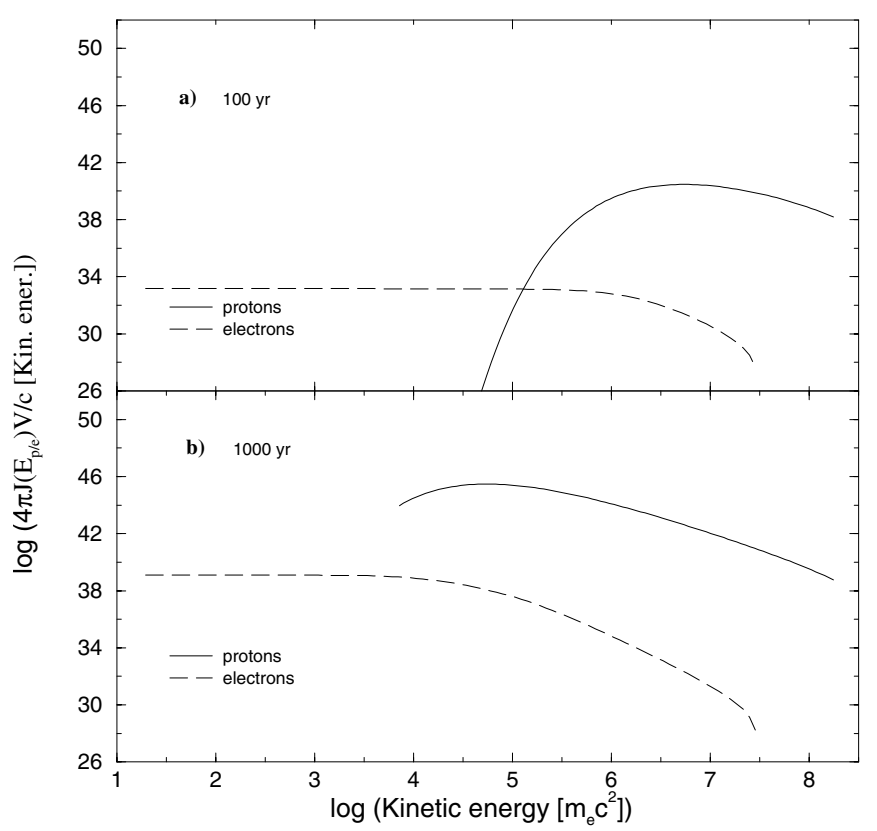

Fig. 3. The same as in Fig. 2 but for a continuous MQ (recall: a) $t=100 \mathrm{yr}$; b) $t=1000 \mathrm{yr}$ ).

proton injection along the MQ lifetime, whereas in the impulsive MQ protons do not accumulate and secondary particles are injected with a changing rate. For longer periods of time, the impulsive MQ emission will eventually disappear, but the continuous MQ will be emitting at least during its whole life. The secondary particles accumulate also in the cloud for the impulsive MQ, but there is no injection of fresh particles at the highest energies, so the spectrum becomes steeper than in the continuous MQ. Below a certain energy, the electron

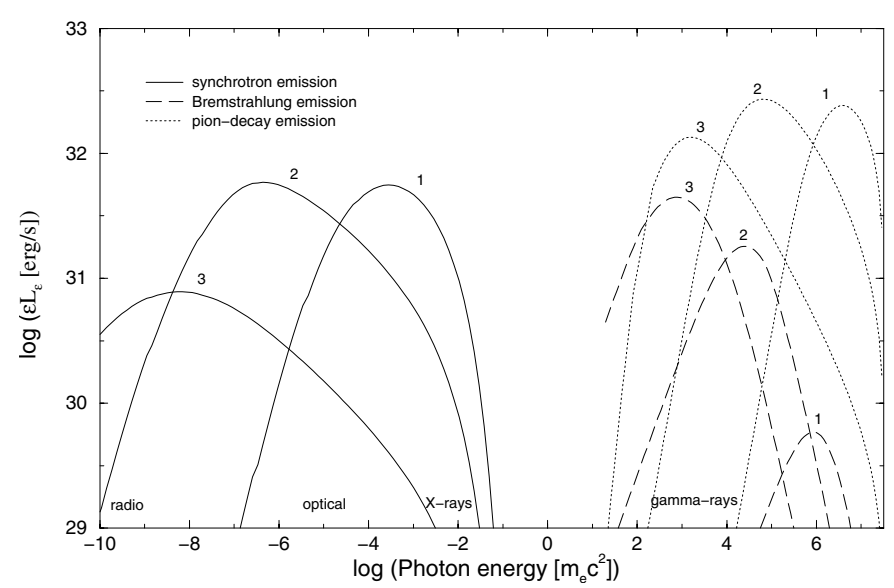

Fig. 4. The SED for an impulsive MQ for $R=10 \mathrm{pc}$ at three different epochs: for $100 \mathrm{yr}$ (curve 1); for $1000 \mathrm{yr}$ (2); and for $10000 \mathrm{yr}$ (3). The photon energy axis units in this and following plots are $m_{\mathrm{e}} c^{2}$.

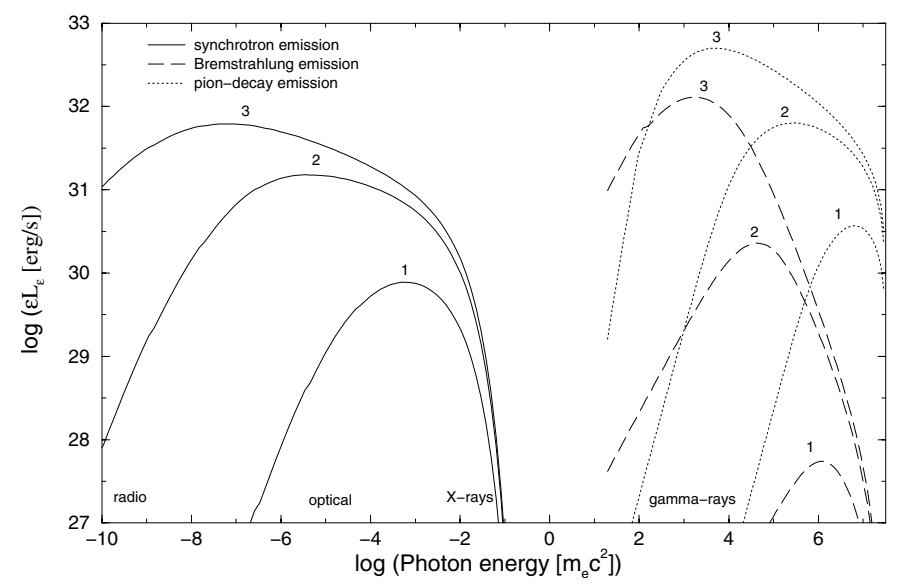

Fig. 5. The same as in Fig. 4 but for a continuous MQ (recall: $t=100 \mathrm{yr}(1) ; 1000 \mathrm{yr}(2)$; and $10000 \mathrm{yr}$ (3)).

energy distribution is completely flat. This comes from both the lower energy cutoff of the proton energy distribution due to the dependence on energy of the diffusion in the ISM and the characteristics of the pp interaction and production of secondary particles.

\subsection{Overall spectrum at different ages}

$R=10 \mathrm{pc} ; t=100,1000,10000 \mathrm{yr}$.

We have computed the SED from radio to $\gamma$-ray energies at different $t$. Both the impulsive and the continuous MQ cases are presented in Figs. 4 and 5, respectively. The luminosity peaks of primary and secondary particle emission are shifted to lower energies as incoming primary particles have less energy and secondary electrons lose their energy. Also, the secondaries accumulate within the cloud (assuming they do not escape), decreasing the difference between the fluxes from the $\pi^{0}$-decay and bremsstrahlung channels.

The comparison of Figs. 4 and 5 shows different spectral features. The spectrum for an impulsive MQ at low and high energies is steeper and presents lower energy luminosity peaks than the one for a continuous source. Also, the total emission 
increases first and decreases later for the first case, increasing asymptotically for the second case. This is due to the lack of continuous injection of new protons at high energies and the reduction of the number of protons at different energies with time for an impulsive MQ. In the continuous source, since protons are continuously arriving at the cloud following the same energy distribution, there are cumulative effects on radiation. The previous issues make the impulsive case a good candidate to be detected at early stages of the source age (100-1000 yr) and at higher energies (i.e. detectable by the new generation of ground-based Cherenkov telescopes), and a good case to determine the energy of the progenitor particles of the emission (due to the weaker cumulative effects of the spectrum). Otherwise, the continuous case should be easier to detect at later stages of the source age (>10000 yr) and at lower energies (i.e. detectable by EGRET), but the cumulative spectral effects would give a bias in the extrapolation of the typical particle energies from the peaks in the spectrum ${ }^{1}$.

\subsection{Spectra for different diffusive behaviors and proton origins}

$R=10 \mathrm{pc}$ (continuous $M Q$ ); steady regime; $E_{\mathrm{pmax}} \sim 10^{6} \mathrm{GeV}$; $p=2.75$ (cosmic rays) and $=2$ (continuous $M Q$ ).

In this subsection, we have computed the overall spectrum assuming that the relativistic protons belong to the sea of cosmic rays. We have computed also the spectra for two continuous MQ cases: an energy-dependent diffusive case and a energy-independent diffusive case. Since the cosmic ray sea can be considered to follow a homogeneous energy distribution in space and time, for this case we have assumed that there is no dependence on energy in the diffusive process, as well as an accelerator age long enough to reach the steady regime. In the second situation, it is possible to compare the energy-dependent diffusion spectrum with the energy independent diffusion spectrum, taking the initial energy distribution of protons to be exactly the same (power-law index, kinetic energy luminosity of the jet, etc.). Again, the age has been taken long enough to reach the steady regime for relativistic protons within the cloud. In Fig. 6, upper panel, the cosmic ray case is shown, the two continuous MQ cases being presented in the lower panel. Since galactic cosmic rays can reach higher energies than the adopted maximum energies for protons, we have now taken $E_{\mathrm{pmax}} \sim 10^{6} \mathrm{GeV}$ in the three SED to compare them properly.

Figure 6 demonstrates the importance of energy-dependent diffusion in obtaining the correct spectral shape. Without energy dependence in the diffusive process, the fluxes grow at all the wavelengths in such a way that there is neither spectral steepening nor emission peak shifting with time. Also, comparing the continuous MQ cases with the cosmic ray one, there is an important difference in the spectral slope between the case with no energy dependence in diffusion and the cosmic ray case, but not between the one with energy dependence in diffusion and the cosmic ray case. In the context of our model,

\footnotetext{
1 Actually, for secondaries, what should be studied is the turning point in the spectrum due to energy losses.
}

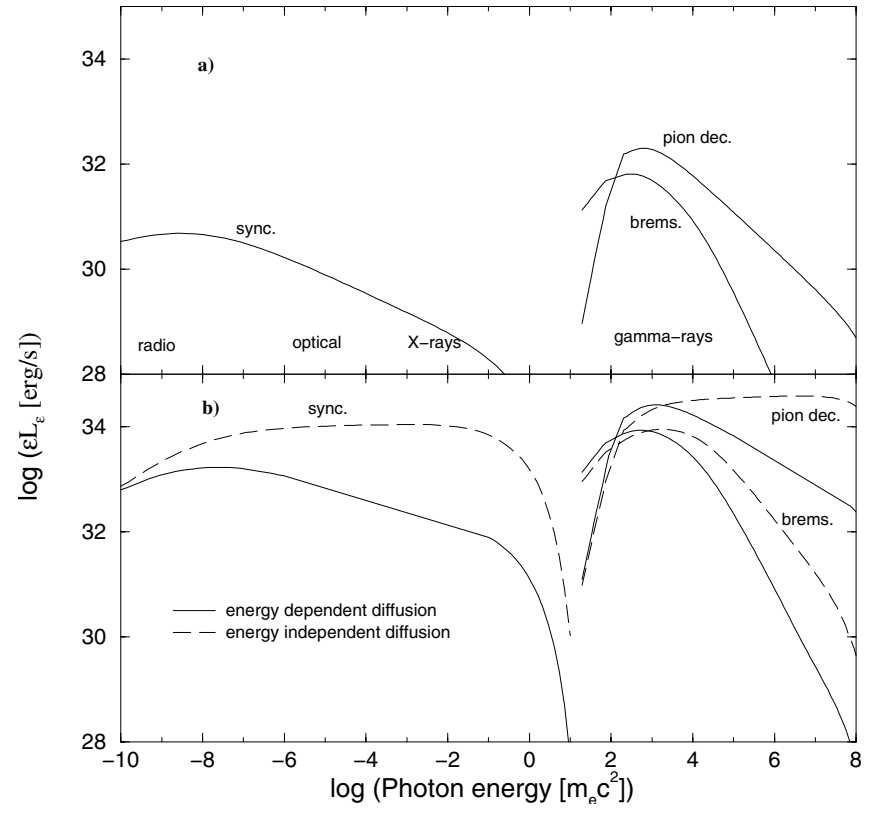

Fig. 6. a) SED from the galactic cosmic rays interacting with a cloud. The power-law index for proton energy distribution has been taken to be 2.75 , as is found near the Earth. Diffusion does not depend on energy. b) SED from a continuous MQ: in one case the diffusion is energy dependent (solid line), in the other case it is not (longdashed line).

it means that the origin of the cosmic ray sea is a group of sources which are far enough from their corresponding interacting clouds to suffer steepening in the proton energy distribution, following these protons initially a power-law of index $\sim 2$ (like the typical value adopted in this work).

\subsection{Overall spectrum at different distances}

\section{$R=10,30,100 \mathrm{pc} ; t=1000 \mathrm{yr}$.}

In Figs. 7 and 8, we present the computed SED from radio to $\gamma$-ray energies of an impulsive and a continuous MQ, respectively. Due to the effects imposed by the propagation of particles in the ISM, there is a relationship between time and distance (see Eq. (4)). This fact implies that Figs. 7 and 8 will present similar features to Figs. 4 and 5, like the decreasing difference in luminosity between $\pi^{0}$-decay and bremsstrahlung emission and the shift of the emission peaks to lower energies.

\subsection{Low and high magnetic fields}

$R=10 ; t=100,10000 \mathrm{yr} ; B=10^{-3}, 10^{-5} \mathrm{G}$.

We have calculated the SED of an impulsive MQ taking a low and a high value for $B$. For a young source case, plotted in Fig. 9, and a low $B$, owing to the small loss rate of secondary particles, the position in energy of the maximum of emission for the secondary particles mainly depends on the typical energy of the arriving protons at that $R$ and $t$. For a young source and a high $B$ (same Fig. 9), we recover the strong dependence of the secondary particles on time due to the losses. Otherwise, for an older source case, plotted in Fig. 10, and a low B, the peak for bremsstrahlung shows at what energy the electrons are 


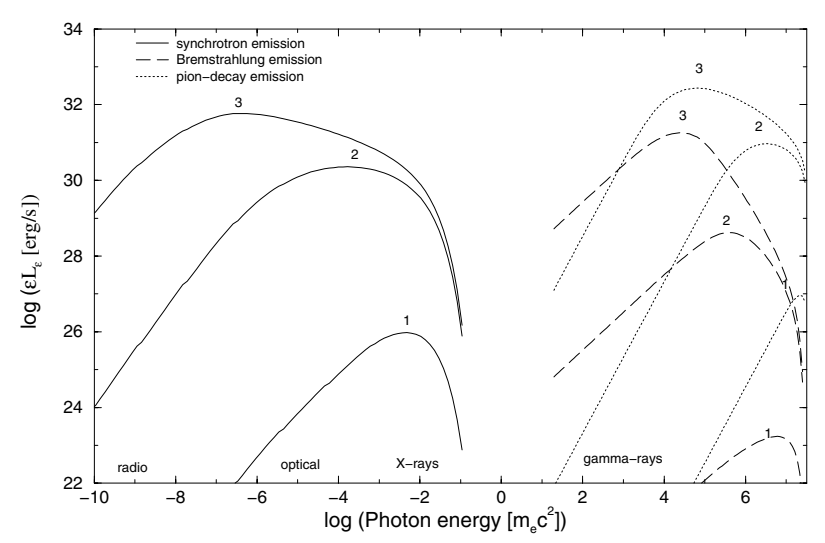

Fig. 7. The SED for an impulsive MQ for $t=1000 \mathrm{yr}$ at three different $R$ : for $100 \mathrm{pc}$ (curve 1), for $30 \mathrm{pc}(2)$, and for $10 \mathrm{pc}(3)$.

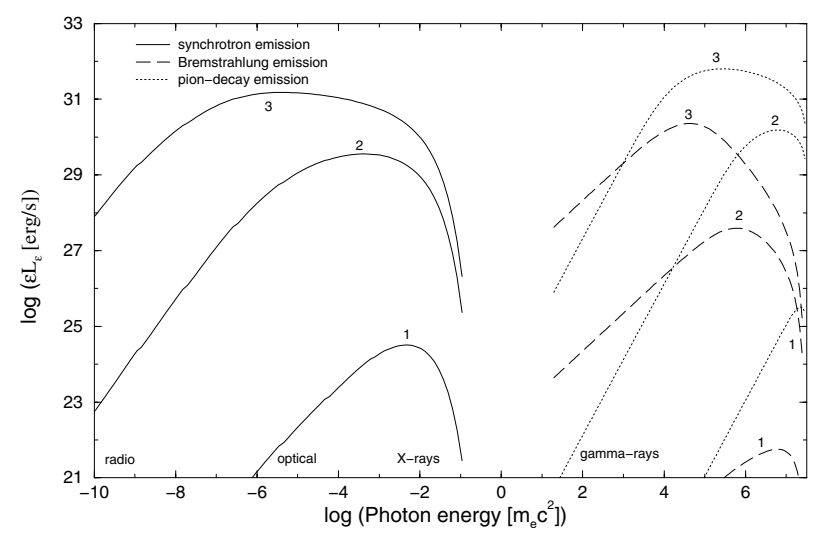

Fig. 8. The same as in Fig. 7 but for a continuous MQ (recall: $R=100 \mathrm{pc}(1), 30 \mathrm{pc}(2)$, and $10 \mathrm{pc}(3))$.

concentrated (roughly the same as protons) although the synchrotron emission peaks at higher energies. For an older source and a high $B$ (same Fig. 10), the computed SED again shows strong dependence of the secondary particles on time due to the losses, presenting a softer spectral slope than in the case of a small $B$ at the same age. In all the previous situations it can be seen how bremsstrahlung and synchrotron are processes more or less important to each other depending on the synchrotron losses: for high $B$, synchrotron dominates, for low $B$, bremsstrahlung dominates. Of course, the magnetic field does not affect $\pi^{0}$-decay emission.

In previous subsections the IC component has not been shown. This is due to the low emission fluxes produced via IC scattering between secondaries and IR photons within the cloud for the adopted density of such photons (see Table 1), much lower than the ones predicted for the other components (synchrotron, bremstrahlung and pion-decay). Nevertheless, in the next subsection, a study about the importance of the IC radiation is presented.

\subsection{On the importance of the IC radiation}

$R=10 ; t=1000 ; U=1,10^{4} \mathrm{eV} \mathrm{cm}^{-3}$.

In Fig. 11 we show the importance of the IC emission comparing with the radiation from other components (synchrotron,

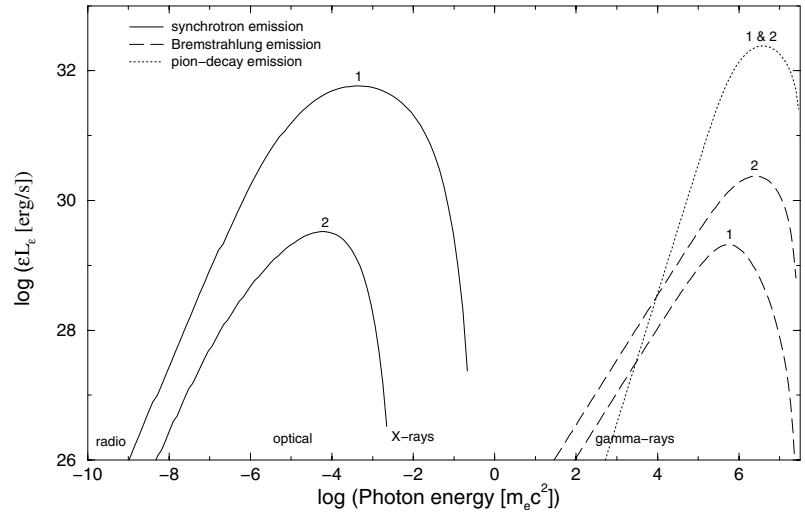

Fig. 9. SED of an impulsive MQ taking $R=10 \mathrm{pc}, t=100 \mathrm{yr}$, and two magnetic field strength $B=10^{-3} \mathrm{G}(1)$ and $10^{-5} \mathrm{G}(2)$.

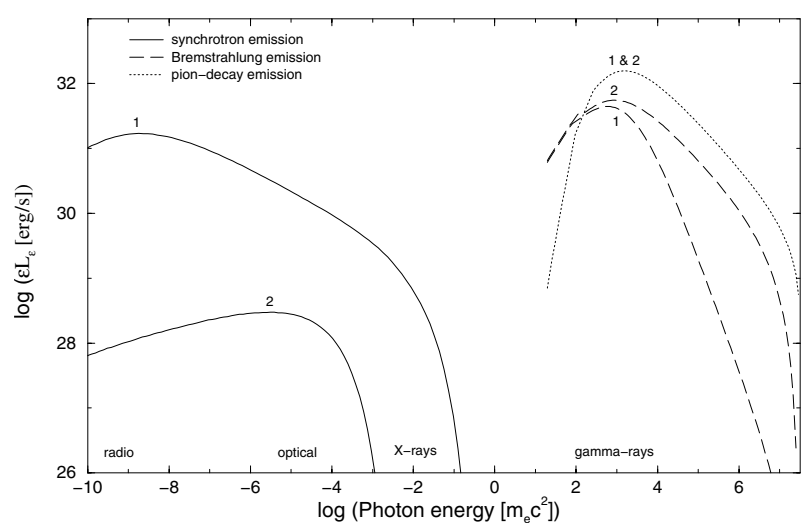

Fig. 10. The same as in Fig. 9, but for a $t=10000 \mathrm{yr}\left(B=10^{-3} \mathrm{G}(1)\right.$ and $\left.10^{-5} \mathrm{G}(2)\right)$.

bremsstrahlung and $\pi^{0}$-decay processes) depending on the energy density of the infrared seed photon field. We recall that the IR spectrum has been taken to be a Planckian grey body, with a temperature of about $50 \mathrm{~K}$. From Fig. 11, it is seen that a high density IR photon field $\left(\geq 10^{4} \mathrm{eV} \mathrm{cm}^{-3}\right)$ is necessary within the cloud to have a relevant IC component in radiation. Thus, for clouds with standard IR photon energy densities, the dominant source of electron energy losses would be the magnetic field, and for clouds with IR photon energy densities about four orders of magnitude higher than the standard value, the dominant source of electron energy losses would be IC scattering, with IC emission levels similar to those generated by the bremsstrahlung process in the same energy range. However, IR field densities of $10^{4} \mathrm{eV} \mathrm{cm}^{-3}$ are too high, such a situation being very unlikely. Of course, $\pi^{0}$-decay emission is not affected by the IR photon field density. It is interesting to note the small decrease of synchrotron and bremsstrahlung emission due to the moderate increase of losses because of the higher radiation field density, compared to the strong increase of IC emission. It seems very unlikely that IC losses could be important enough to control by themselves the radiative processes and the evolution of secondary particles in a reasonable scenario. 


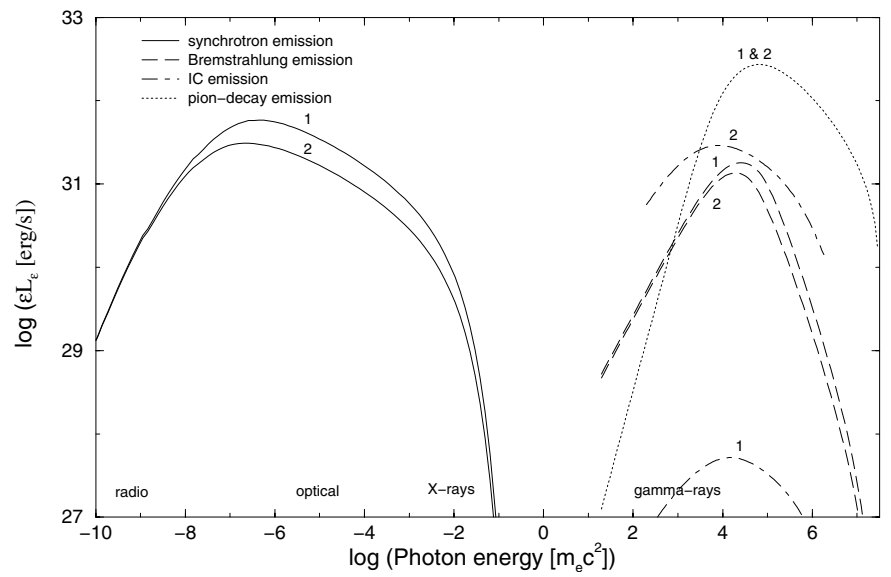

Fig. 11. SED of an impulsive source at $R=10 \mathrm{pc}$ after $t=1000 \mathrm{yr}$. We have plotted both the cases for $U=1 \mathrm{eV} \mathrm{cm}^{-3}$ (1) and $U=10000 \mathrm{eV} \mathrm{cm}^{-3}$ (2).

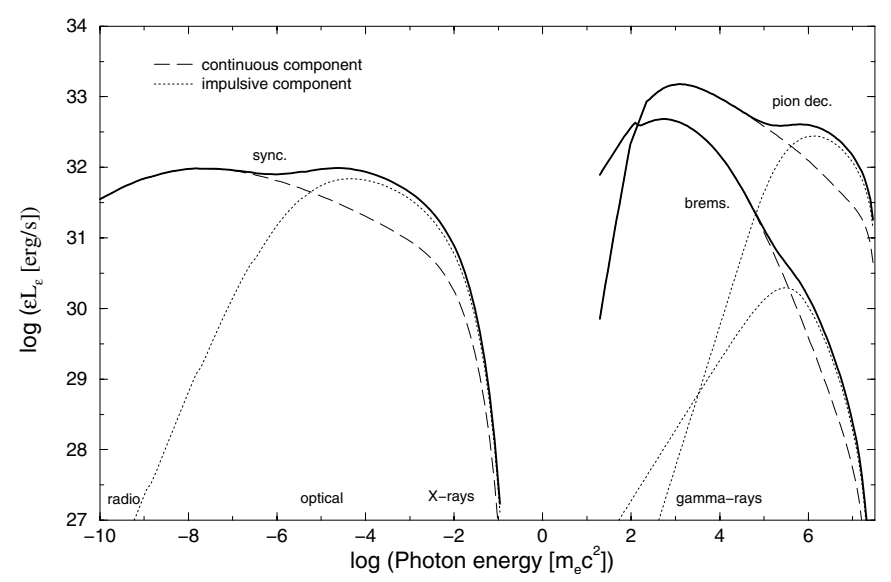

Fig. 12. SED of a complex source: a continuous MQ (longdashed line) plus an impulsive phase (dotted line). $t=10^{5} \mathrm{yr}$ for the first one and $t=200 \mathrm{yr}$ for the second one, with $R=10 \mathrm{pc}$. The added spectrum is presented as a thick solid line. The kinetic energy luminosity for the continuous MQ has been taken to be $10^{37} \mathrm{erg} \mathrm{s}^{-1}$, and the kinetic energy for the impulsive MQ has been taken to be $10^{48} \mathrm{erg}$.

\subsection{Continuous $M Q$ with a strong outburst}

$R=10 \mathrm{pc} ; t=10^{5} \mathrm{yr}$ (continuous $\left.M Q\right)$ and $t=200 \mathrm{yr}$ (impulsive $M Q)$.

Now we combine two kind of sources, an impulsive MQ and a continuous MQ, to obtain the complex SED that is presented in Fig. 12. To find clouds presenting emission with different origins is quite probable. Taking into account the longterm variable nature of microquasars (the short-term is not taken into account here because it does not affect large-scale objects like clouds), we can find different periods of activity such as persistent and impulsive phases. Therefore, a spectrum like the one shown in Fig. 12 might be quite typical. Due to obvious differences in evolution for both types of emission, that originating in the continuous phase and that originating in the impulsive phase, we find that the spectral shape presents two different peaks and complicated slopes, being detectable at radio, X-ray, EGRET and TeV enegies.

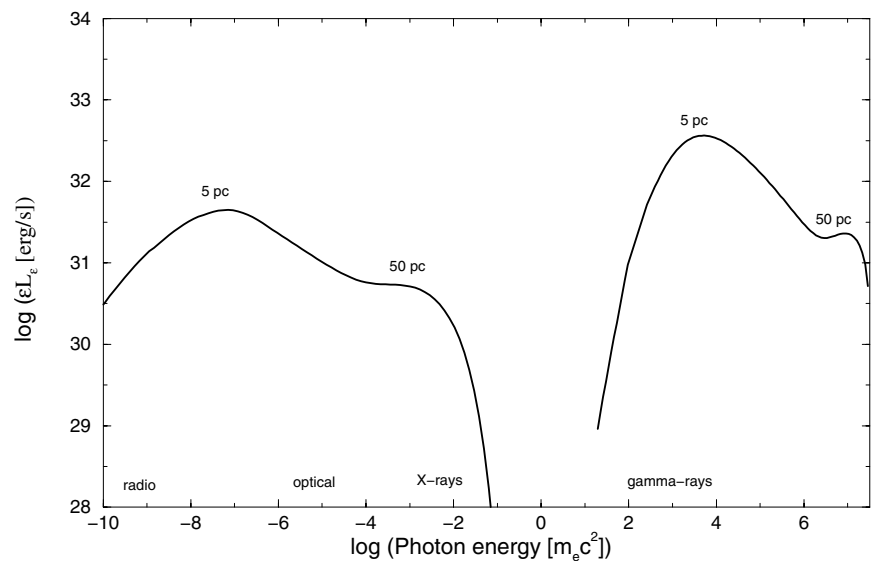

Fig. 13. SED of the observed spectrum from two clouds interacting with an impulsive MQ at distances 5 and $50 \mathrm{pc}$.

\subsection{The case of two clouds}

$R=5 \mathrm{pc}$ and $R=50 \mathrm{pc}$, with $V \sim 3.4$ and $3400 \mathrm{pc}^{3}$, respectively.

In Fig. 13 we have computed the spectrum that would be observed if there were two clouds at different known distances from the origin of the particles. We assume an impulsive MQ as being more clear than the continuous one to determine its physical parameter values due to the reduced particle accumulation effects. All the other parameters except the distance from the clouds to the MQ would be unknown a priori, and our task is to estimate them from the observed spectrum using our model.

From the characteristic frequency of the synchrotron emission $\left(v_{\mathrm{c}}=6.3 \times 10^{18} B E_{\mathrm{e}}^{2}\right)$ and the maximum energy reached by both the synchrotron and the $\pi^{0}$-decay emissions, we can roughly determine the magnetic field within the clouds $\left(5 \times 10^{-4} \mathrm{G}\right)$ and the electron energy at the peaks and turning points. In addition, Eqs. (4) and (8) can help to estimate the time of travel for the first particles from the MQ to the cloud and the time during which the secondary particles have evolved (the total age, $t=1000 \mathrm{yr}$ ). From previous parameters, we estimate the diffusion coefficient constant $\left(D_{10}=10^{27} \mathrm{~cm}^{2} \mathrm{~s}^{-1}\right)$. The spectral shape can also give information about the proton energy distribution. Knowing the previous set of parameter values and the volume of the cloud, it could be possible to find the remaining parameters: $n_{\mathrm{H}}$ and the total kinetic energy of the released protons.

\section{MQ, clouds and non variable $\gamma$-ray sources}

Statistical studies of the EGRET sources in the galactic plane point to the association between these sources and high density regions in the inner spiral arms including star formation regions and molecular clouds (Romero et al. 1999; Bhattacharya et al. 2003). It has been claimed that the low latitude EGRET sources can be divided in two different subgroups, one formed by those sources that show variability, and another formed by dubious or steady cases (Romero et al. 2004). High-mass MQs have been proposed as possible variable EGRET source counterparts (Kaufman Bernadó et al. 2002; Bosch-Ramon et al. 2005). The results of this paper show that MQs could be indirectly 


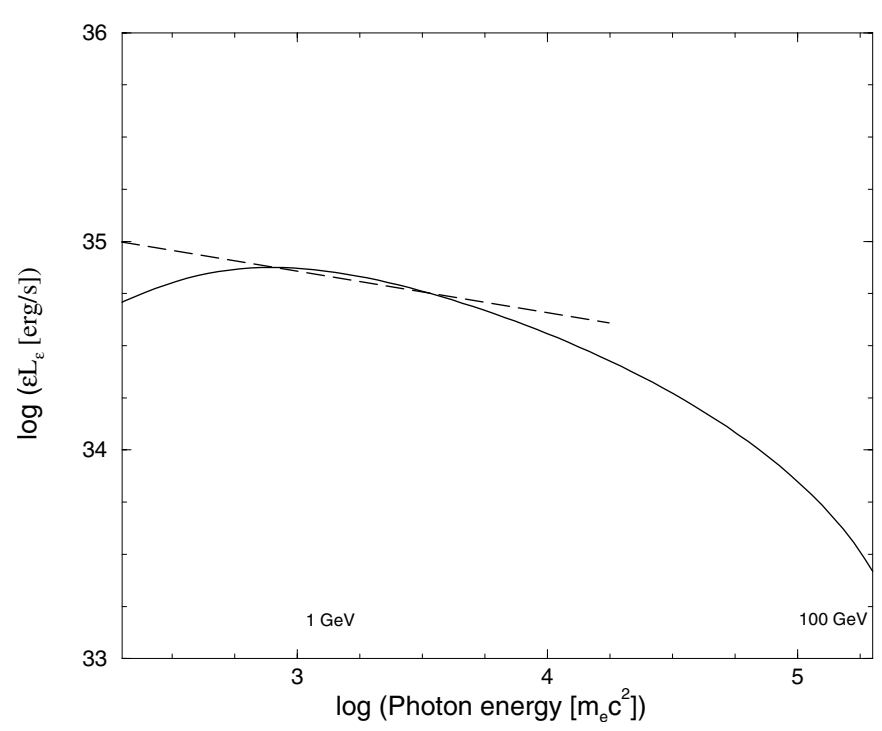

Fig. 14. SED of a continuous MQ at $t=10^{6} \mathrm{yr}$ and $R=10 \mathrm{pc} \mathrm{re}-$ producing a typical $\mathrm{GeV}$ source (solid line). The value of the cloud mass is $10^{5} \mathrm{M}_{\odot}$. An extrapolation of a typical EGRET spectrum up to $\sim 10 \mathrm{GeV}$ is also shown (long-dashed line).

responsible for some of the non-variable EGRET sources. Taking into account that our model can reproduce a typical EGRET spectrum (see Fig. 14) of a steady source, and the association between high-mass MQs and high density regions, the scenario contemplated here could be the explanation of, at least, a significant fraction of the non variable high-energy $\gamma$-ray detections. In addition, these sources could produce detectable emission at $\mathrm{TeV}$ energies.

In Fig. 14, we show the predicted SED produced by the interaction of protons released from a persistent jet of a MQ and hydrogen nuclei of a giant molecular cloud of $10^{5} M_{\odot}$ at $R=10 \mathrm{pc}$ and $t=10^{6} \mathrm{yr}$. At a typical Galactic distance, say $4 \mathrm{kpc}$, we obtain computed levels of emission in the EGRET range similar to the ones observed by this instrument, with spectral slopes similar to the average value $(\sim 2.16)$ of this steady subgroup of EGRET sources of the galactic plane (see Bosch-Ramon et al. 2005). These points make it reasonable to suggest an indirect association between the steady EGRET population and MQs. We note that, due to the small distance between the cloud and the MQ considered here in comparison to the cloud size, our results are a first order approach and an inhomogeneous model is required to improve the accuracy of our predictions, which will be presented in a future work.

MQs can emit $\gamma$-rays by themselves (i.e. Bosch-Ramon et al. 2005). The $\gamma$-ray emitting MQ and cloud could have an angular separation too small to be distinguished by EGRET (although they could be distinguishable at lower energies). In such a case, the stronger emitter of $\gamma$-rays would dominate the spectrum and variability properties and the next generation $\gamma$-ray instruments, with better angular resolution than EGRET, would be required to separate them.

One interesting issue is the shape of the young proton spectra (see Fig. 1) and the subsequent electron energy distributions (see Figs. 2 and 3). As is seen from the mentioned plots, there is a natural low-energy cutoff in the proton spectrum produced by the energy-dependent diffusion, which implies an almost monoenergetic distribution of electrons peaking at high energies. This characteristic of the secondary particle energy distribution has a clear implication for the related synchrotron spectrum: a slope like the one presented by a monoenergetic electron spectrum at low frequencies with a spectral index of $1 / 3$. Therefore, in the context of a MQ interacting with a cloud, we expect to observe extended and steady emission with a spectral index of $1 / 3$ at radio and even shorter wavelengths. For instance, detected radiation coming from the region near the galactic center shows a similar spectrum (see, i.e., Lesch et al. 1988), possibly generated in a scenario like the one proposed here.

\section{Conclusions}

The study of the emission coming from high density regions of the ISM provides us with information about accelerators of high energy particles located near these clouds. In this work, we have explored the implications of the presence of a MQ close to a cloud. The obtained results predict that extended radiation from radio frequencies to soft X-rays, generated by synchrotron radiation, could be detected from clouds, together with $\mathrm{GeV}$ and $\mathrm{TeV} \gamma$-rays produced mainly via $\pi^{0}$ decay. Further information can be obtained about cloud internal characteristics (average magnetic fields, average densities). If the jets of MQs are sources of relativistic hadrons, then nearby clouds can be indicators of such hadronic accelerators with a specific broadband spectrum from radio to very-high energy $\gamma$-rays.

Acknowledgements. We thank Diego Torres for useful comments. We also thank anonymous referee for constructive comments and suggestions. V.B.-R. and J.M.P. acknowledge partial support by DGI of the Ministerio de Ciencia y Tecnología (Spain) under grant AYA-2001-3092, as well as additional support from the European Regional Development Fund (ERDF/FEDER). During this work, V.B.-R. has been supported by the DGI of the Ministerio de Ciencia y Tecnología (Spain) under the fellowship FP-2001-2699.

\section{References}

Aharonian, F. A., \& Atoyan, A. M. 1996, A\&A, 309, 917 Aharonian, F. A., \& Atoyan, A. M. 2000, A\&A, 362, 937

Aharonian, F. A., Belyanin, A. A., Derishev, E. V., Kocharovsky, V. V., \& Kocharovsky, Vl. V. 2002, Phys. Rev. D, 66, 3005

Atoyan, A. M., Aharonian, F. A., \& Völk, H. J. 1995, Phys. Rev. D, 52,3265

Bhattacharya, D., Akyüz, A., Miyagi, T., Samimi, J., \& Zych, A. 2003, A\&A, 404, 163

Blumenthal, G. R., \& Gould, R. J. 1970, Rev. Mod. Phys., 42, 237

Bosch-Ramon V., Romero, G. E., \& Paredes, J. M. 2005, A\&A, 429, 267

Corbel, S., Fender, R. P., Tzioumis, A. K., Tomsick, J. A., et al. 2002, Science, 298, 196

Ginzburg, V. L., \& Syrovatskii, S. I. 1964, The Origin of Cosmic Rays (New York: Macmillan), 1964

Gliozzi, M., Bodo, G., \& Ghisellini, G. 1999, MNRAS, 303, 37

Heinz, S. 2002, A\&A, 388, L40

Heinz, S., \& Sunyaev, R. 2002, A\&A, 390, 751

Kaaret, P., Corbel, S., Tomsick, J. A., et al. 2003, ApJ, 582, 945 
Kaufman Bernadó, M. M., Romero, G. E., \& Mirabel, I. F. 2002, A\&A, 385, L10

Lesch, H., Schlickeiser, R., \& Crusius, A. 1988, A\&A, 200, L9

Marshall, H. L., Canizares, C. R., \& Schulz, N. S. 2002, ApJ, 564, 941

Martí, J., Paredes, J. M., \& Peracaula, M. 2000, ApJ, 545, 939

Meier, D. L. 2003, New Astron. Rev., 47, 667

Mirabel, I. F., \& Rodríguez, L. F. 1994, Nature, 371, 4

Mirabel, I. F., \& Rodríguez, L. F. 1999, ARA\&A, 37, 409

Nolan, P. L., Tompkins, W. F., Grenier, I. A., \& Michelson, P. F. 2003, ApJ, 597, 615

Paredes, J. M., Martí, J., Ribó, M., \& Massi, M. 2000, Science, 288, 2340

Paredes, J. M., Ribó, M., Ros, E., Martí, J., \& Massi, M. 2002, A\&A, 393, L99
Ribó, M. 2002, Ph.D. Thesis, Universitat de Barcelona

Romero, G. E., Benaglia, P., \& Torres, D. F. 1999, A\&A, 348, 868

Romero, G. E., Torres, D. F., Kaufman Bernadó, M. M., \& Mirabel, I. F. 2003, A\&A, 410, L1

Romero, G. E., Grenier, I. A., Kaufman Bernadó, M. M., Mirabel, I. F., \& Torres, D. F. 2004, ESA-SP, 552, 703

Spencer, R. E. 1984, MNRAS, 209, 869

Tauris, T. M., \& van den Heuvel, E. 2003, Compact Stellar X-Ray Sources, ed. W. H. G. Lewin, \& M. van der Klis (Cambridge University Press)

Torres, D. F., Romero, G. E., Dame, T. M., Combi, J. A., \& Butt, Y. M. 2003, Phys. Rep., 382, 303

Wang, X. Y., Dai, Z. G., \& Lu, T. 2003, ApJ, 592, 347 